\title{
Comprehensive Study on Investigation on Topology of the Line and Plane
}

\author{
Khin Khin Aye \\ Lecturer \\ Department of Engineering Mathematics \\ Technological University (Hmawbi), Yangon, Myanmar
}

\begin{abstract}
In this paper we have discussed the definitions and the basic properties of open sets, closed sets, accumulation points or limit points and sequence. Sets may be neither open nor closed. The reader should not confuse the concept "limit point of a set" with the different, though related, concept "limit of a sequence". Some of the solved and supplementary problems will show the relationship between these two concepts. Observe that $\left\langle a_{n}: n \in N\right\rangle$ denotes a sequence and is a function. On the other hand, $\left\{a_{n}: n \in N\right\}$ denotes the range of the sequence and is a set. We have given several characterizations of these sets. We discuss the definitions of bounded sequence, convergent sequence, Cauchy sequence and their relations. Then we can study every bounded sequences of real numbers contains a convergent subsequence and every Cauchy sequence of real numbers converges to a real number. Let A be a bounded, infinite set of real numbers. Then A has at least one accumulation point. We express the definition of topology, usual topology and topological space. The creation of topology the science of spaces and figures that remains unchanged under continuous deformations represents a phenomenon of this kind, but of a distinctly modern variety. Then we begin our study of some properties topological spaces by making the idea of being connected that is being in one piece. We observe that, for the usual topology on the line $R$ and in the plane $R^{2}$. Finally we express the characterizations of the discrete topological space and indiscrete topological space.
\end{abstract}

Key Words: Sets, Sequences, Topology, Topological Spaces.

\section{INTRODUCTION}

This paper provides the discussion in three sections. The first one is the basis definitions of open sets and their properties on topology on the line $\mathrm{R}$ and in the plane $\mathrm{R}^{2}$. The second is the basis definitions of closed sets and their properties on topology on the line $\mathrm{R}$ and in the plane $\mathrm{R}^{2}$. And finally, we study the basis properties of topological spaces.

The set of real numbers, denotes by R, plays a dominant role in mathematics and in particular, in analysis. In fact, many concepts in topology are abstractions of properties of set of real numbers. We assume the reader is familiar with the geometric representation of $\mathrm{R}$ by means of the points on a straight line and in the plane, by means of the points on a plane [1].

\section{BASIS PROPERTIES}

\subsection{Open Sets}

Let $A$ be a subset of R. A point $p \in A$ is an interior point of $A$ if and only if $p \in$ some open interval $S_{p}$ which is contained in $A ; p \in S_{p} \subset A$. The set $A$ is open if and only if each of its points is an interior point [1].

\subsubsection{Example}

(i) An open interval $A=(a, b)$ is an open set. For, we may choose $S_{p}=A$ for each $p \in A$.

(ii) The real line $\mathrm{R}$, itself, is open since any open interval $S_{p}$ must be a subset of $\mathrm{R}$, that is $p \in S_{p} \subset R$.

(iii) The closed interval $B=[a, b]$ is not an open set.

For, any open interval containing $a$ or $b$ must contain points outside of $B$. Hence the end points $a$ and $b$ are not interior points of $B$.

(iv) The empty set $\emptyset$ is open since there is no point in $\emptyset$ which is not an interior point.

(v) The infinite open intervals, i.e., the subsets of $\mathrm{R}$ defined and denoted by $(a, \infty)=\{x \mid x \in R, x>a\},(-\infty, a)=\{x \mid x \in R, x<a\}$ and $(-\infty, \infty)=\{x \mid x \in R\}=R$ are open sets.

On the otherhand, the infinite closed intervals, i.e., the subsets of $\mathrm{R}$ defined and denoted by $[a, \infty)=\{x \mid x \in R, x \geq a\}$, $(-\infty, a]=\{x \mid x \in R, x \leq a\}$ are not open sets since $a \in R$ is not an interior point of either $[a, \infty)$ or $(-\infty, a]$. 
International Journal of Advances in Scientific Research and Engineering (ijasre), Vol 5 (12), December-2019

\subsubsection{Theorem}

The union of any number of open sets in $\mathrm{R}$ is open.

\subsubsection{Theorem}

The intersection of any finite number of open sets in $\mathrm{R}$ is open.

Next, we will show that an arbitrary intersection of open sets need not be open.

\subsubsection{Example}

Let $A_{n}=\left\{\left(-\frac{1}{n}, \frac{1}{n}\right) \mid n \in N\right\}$ be the class of open intervals. Show that $\bigcap_{n=1}^{\infty} A_{n}$ is not an open set.

Solution

$A_{n}=\left\{\left(-\frac{1}{n}, \frac{1}{n}\right) \mid n \in N\right\}$, i.e., $\left\{(-1,1),\left(-\frac{1}{2}, \frac{1}{2}\right),\left(-\frac{1}{3}, \frac{1}{3}\right), \ldots\right\}$.

The intersection $\cap_{n=1}^{\infty} A_{n}=\{0\}$ of the open intervals consists of the single point 0 which is not an open set.

Hence an arbitrary intersection of open sets is not an open set.

\subsection{Open Disc}

A open disc $D$ in the plane $R^{2}$ is the set of points inside a circle, with center $p=\left\langle a_{1}, a_{2}\right\rangle$ and radius $>0$, i.e.,

$D=\left\{q \in R^{2}: d(p, q)<\delta\right\}$, where $d(p, q)$ denotes the usual distance between two points $p=\left\langle a_{1}, a_{2}\right\rangle$ and $q=\left\langle b_{1}, b_{2}\right\rangle$ in $R^{2}$.

Let $A$ be a subset of $R^{2}$. A point plane $p \in A$ is an interior point of $A$ if and only if $p$ belongs to some open disc $D_{p}$ which is contained in $A: p \in D_{p} \subset A$.

The set $A$ is open if and only if each of its points is an interior point [1-2].

\subsubsection{Example}

Show that the union of any number of open subsets of $R^{2}$ is open.

Solution

Let $A$ be a class of open subsets of $R^{2}$.

Let $H=\bigcup\{G: G \in A\}$.

Let $p \in H$.

We must show that $p$ is an interior point of $H$.

Since $p \in H, \exists G_{0} \in A: p \in G_{0}$.

But $G_{0}$ is an open set, hence there exists an open disc $D_{p}$ such that $p \in D_{p} \subset G_{0} . G_{0} \subset H$ and so $D_{p} \subset$ H.i.e.p $\in D_{p} \subset H$.

Hence $p$ is an interior point of $H$ and so $H=U G$ is open set.

\subsubsection{Example}

Show that the intersection of any finite number of open subsets of $R^{2}$ is open.

Solution

Let $G$ and $H$ be open subsets of $R^{2}$.

To prove, $G \cap H$ is open.(i.e., $p \in S_{p} \subset G \cap H$ ).

Let $p \in G \cap H$.

Then $p \in G$ and $p \in H$.

Since $G$ and $H$ are open sets, there exists open disc $D_{1}$ and $D_{2}$ such that $p \in D_{1} \subset G$ and $p \in D_{2} \subset H$.

Then $p \in D_{1} \cap D_{2} \subset G \cap H$.

But $D_{1} \cap D_{2}=D$.

Therefore $p$ is an interior point of $G \cap H$.

Hence $G \cap H$ is open.

\subsubsection{Example}

Prove that every open subset $G$ of the plane $R^{2}$ is the union of open discs.

Solution

Since $G$ is open, for each $p \in G$ there is open disc $D_{p}$ such that $p \in D_{p} \subset G$.

Then $G=\cup\left\{D_{p} \mid p \in G\right\}$.

\subsubsection{Example}

Let $G$ be an open subset of $R^{2}, p \in G$. Prove that there exists an open disc $D$ with center $p$ such that $p \in D \subset G$.

Solution

By definition of an interior point, there exists an open disc, $D_{1}=\left\{q \in R^{2}: d\left(p_{1}, q\right)<\delta\right\}$ with center $p_{1}$ and radius $\delta$ such that $p \in D_{1} \subset G$. So $d\left(p_{1}, p\right)<\delta$.

Let $r=\delta-d\left(p_{1}, p\right)>0$ and $D=\left\{q \in R^{2}: d(p, q)<\frac{r}{2}\right\}$.

Then $p \in D \subset D_{1} \subset G$, where $D$ is an open disc with center $p$.

\subsection{Accumulation Point or Limit Point}

Let $A$ be a subset of $R$. A point $p \in R$ is an accumulation point or limit point of $A$ if and only if every open set $G$ containing $p$ 
contains a point of $A$ different from $p$; i.e., $G$ open, $p \in G$ implies $A \cap(G \backslash\{p\}) \neq \emptyset$.

The set of accumulation points of $A$, denoted by $A^{\prime}$, is called the derived set of $A .[1-3]$.

\subsubsection{Example}

(i) Let $A=\left\{1, \frac{1}{2}, \frac{1}{3}, \frac{1}{4}, \ldots\right\}$.

The point " 0 " is an accumulation point of $A$ since every open set $G$ with $0 \in G$ contains an open invertal $\left(-a_{1}, a_{2}\right) \subset G$ with $-a_{1}<0<a_{2}$ which contains points of $A$. The limits point 0 of $A$ dose not belong to $A$ and $A$ does not contain any other limit points. Hence $A^{\prime}=\{0\}$.

(ii) Let $Q$ be the set of rational numbers.

Every real number $p \in R$ is a limit point of $Q$ since every open set contains rational numbers.

Hence $Q^{\prime}=R$.

(iii) The set of integers $Z=\{\ldots,-2,-1,0,1,2, \ldots\}$.

Then $Z$ does not have any points of accumulation. Hence $Z^{\prime}=\emptyset$.

\subsubsection{Example}

Determine the accumulation points of each set of real numbers: (i) $N$, (ii) $(a, b]$, (iii) $Q^{c}$, the set of irrational points.

Solution

(i) $\quad N=$ the sets of natural numbers. $N$ does not have any limit points.

For, if "a" is any real number, we can find $\delta>0$ such that open set $(\mathrm{a}-\delta, a+\delta)$ contains no point of $N$ other than "a". Hence $N^{\prime}=\emptyset$.

(ii) $(\mathrm{a}, \mathrm{b}]$.

Every point $p \in[a, b]$ is a limit point of $(\mathrm{a}, \mathrm{b}]$.

Since every open interval containing $p \in[a, b]$ will contain points of $(\mathrm{a}, \mathrm{b}]$ other than $p$.

Hence $(a, b]^{\prime}=[a, b]$.

(iii) $Q^{c}$, the set of irrational points.

Every real number $p \in R$ is a limit point of $Q^{c}$ since every open interval containing $p \in R$

Will contain points of $Q^{c}$ other than $p$.

Hence $\left(Q^{c}\right)^{\prime}=R$.

\subsubsection{Example}

Let $A^{\prime}$ be the derived set, i.e., the set of limit points of a set $A$. Find sets $A$ such that

(i) A and $A^{\prime}$ are disjoint,

(ii) $A$ is a proper subset of $A^{\prime}$,

(iii) $A^{\prime}$ is a proper subset of $A$,

(iv) $A^{\prime}=A$.

Solution

(i) Let $A=\left\{1, \frac{1}{2}, \frac{1}{3}, \frac{1}{4}, \ldots\right\}$.

Then $A^{\prime}=\{0\}$ and so $A$ and $A^{\prime}$ are disjoint.

(ii) Let $A=(a, b]$.

Then $A^{\prime}=[a, b]$ and so $A \subset A^{\prime}, A \neq A^{\prime}$.

Hence $A$ is a proper subset of $A^{\prime}$.

(iii) Let $A=\left\{0,1, \frac{1}{2}, \frac{1}{3}, \frac{1}{4}, \ldots\right\}$.

Then $A^{\prime}=\{0\}$ and so $A \subset A^{\prime}, A \neq A^{\prime}$.

Hence $A^{\prime}$ is a proper subset of $A$.

(iv) Let $A=[a, b]$.

Then each point of $A$ is a limit of $A$.

So $A^{\prime}=[a, b]$.

Hence $A=A^{\prime}$.

\subsection{Closed Sets}

A subset $A$ of $R$ is a closed set if and only if its complement $A^{c}$ is an open set [4].

\subsubsection{Theorem}

A subset $A$ of $R$ is a closed if and only if $A$ contains each of its points of accumulation.

\subsubsection{Example}

(i) The closed interval $[\mathrm{a}, \mathrm{b}]$ is a closed set since its complement $(-\infty, a) \cup(b, \infty)$, the union of two open infinite intervals, is open.

(ii) The set $A=\left\{1, \frac{1}{2}, \frac{1}{3}, \frac{1}{4}, \ldots\right\}$ is not closed since 0 is a limit point of $A$ but $0 \notin A$. 
(iii) The empty set $\emptyset$ and the entire line $R$ are closed sets since their complements $R$ and $\emptyset$, respectively, are open sets.

\subsubsection{Example}

A set $F$ is closed if and only if its complement $F^{c}$ is open.

Solution

Since $\left(F^{c}\right)^{c}=F$; So $F$ is the complement of $F^{c}$. Thus, by definition, $F$ is closed if and only if $F^{c}$ is open.

\subsubsection{Example}

Prove that the union of a finite number of closed sets is closed.

Proof

Let $F_{1, \ldots,} F_{m}$ be closed sets and $F=F_{1} \cup \ldots \cup F_{m}$

Then $F=\left(F_{1} \cup \ldots \cup F_{m}\right)^{c}$

$$
=F_{1}{ }^{c} \cap \ldots \cap F_{m}{ }^{c} \text {. }
$$

$F_{i}$ is closed and $F_{i}^{c}$ is open.

So $F^{c}$ is the intersection of a finite number of open sets $F_{i}{ }^{c}$.

Thus $F^{c}$ is also open.

Hence $\left(F^{c}\right)^{c}=F$ is closed.

\subsubsection{Example}

Proved that the intersection of any number of closed sets is closed.

Solution

Let $\left\{F_{i}\right\}$ be a class of closed sets and $F=\bigcap_{i} F_{i}$.

Then $F^{c}=\left(\bigcap_{i} F_{i}\right)^{c}$

$$
F^{c}=\bigcup_{i} F_{i}^{c} \text {. }
$$

$F_{i}$ is closed and $F_{i}{ }^{c}$ is open.

So $F^{c}$ is the union of any number of open sets $F_{i}{ }^{c}$.

Thus $F^{c}$ is open.

Hence $\left(F^{c}\right)^{c}=F$ is closed.

\subsubsection{Example}

Proved that a subset of $R^{2}$ is closed if and only if it contains each of its accumulation points.

Solution

Suppose $p$ is a limit point of a closed set $F \subset R^{2}$. Then every open disc containing $p$ contains points of $F$ other than $p$. Hence there cannot be open disc $D_{p}$ containing $p$ which is completely contained in $F^{c}$.

So $p$ is not an interior point of $F^{c}$. But $F^{c}$ is open, since $F$ is closed.

Then $p \notin F^{c}, p \in F$.

Conversely, suppose $F$ contains each of its accumulation points.

Let $p \in F^{c}$.

Then $p$ is not a limit point of $F$.

Hence there exists at least one open $\operatorname{disc} D_{p}$ containing $p$ such that $D_{p}$ does not contain any points of $F$.

So $D_{p} \subset F^{c}$.

Hence $p$ is an interior point of $F^{c}$ and $F^{c}$ is open. Then $F$ is closed.

\subsection{Sequence}

A sequence denoted by $\left\langle S_{n}\right\rangle=\left\langle s_{1}, s_{2}, s_{3}, \ldots, s_{n}, \ldots\right\rangle, n \in N$ is a function whose domain is $N=\{1,2, \ldots\}$.

The image $S_{n}, n \in N$ is called the $n^{\text {th }}$ term of the sequence. [4]

\subsection{Bounded}

A sequence $\left\langle S_{n}\right\rangle$ is said to be bounded if its range $\left\{S_{n}: n \in N\right\}$ is a bounded set.

\subsubsection{Example}

(i) The sequence $\left\langle S_{n}\right\rangle=\langle 1,3,5, \ldots\rangle$ is not a bounded sequence.

For, The range of $\left\langle S_{n}\right\rangle=\{1,3,5, \ldots\}$ is not bounded and so $\left\langle S_{n}\right\rangle$ is not bounded.

(ii) The sequence $\left\langle S_{n}\right\rangle=\left\langle-\frac{1}{2}, \frac{1}{4},-\frac{1}{8}, \frac{1}{16} \ldots\right\rangle$ is a bounded sequence.

For, The range of $\left\langle S_{n}\right\rangle=\left\{-\frac{1}{2}, \frac{1}{4},-\frac{1}{8}, \frac{1}{16} \ldots\right\}$ is bounded and so $\left\langle S_{n}\right\rangle$ is bounded.

(iii) The sequence $\left\langle S_{n}\right\rangle=\langle 1,0,1,0, \ldots\rangle$ is a bounded sequence.

For, The range of $\left\langle S_{n}\right\rangle=\{0,1\}$ is bounded and so $\left\langle S_{n}\right\rangle$ is a bounded sequence.

\subsection{Converges}

The sequence $\left\langle a_{n}: n \in N\right\rangle$ of real numbers converges to $b \in R$, or, equivalently $b$ the limit of the sequence $\left\langle a_{n}: n \in N\right\rangle$ if for every $\varepsilon>0$ there exists a positive integer $n_{0}$ such that $n>n_{0}$ implies $\left|a_{n}-b\right|<\varepsilon$. 
International Journal of Advances in Scientific Research and Engineering (ijasre), Vol 5 (12), December-2019

\subsection{Cauchy Sequence}

A sequence $\left\langle a_{n}: n \in N\right\rangle$ of real numbers is a Cauchy sequence if and only if for every $\varepsilon>0$ there exists a positive integer $n_{0}$ such that $n, m>n_{0}$ implies $\left|a_{n}-a_{m}\right|<\varepsilon$.In other words, a sequence is a Cauchy sequence if and only if the terms of sequence become arbitrarily close to each other as gets $n$ large [4].

\subsubsection{Example}

Show that every convergent sequence is a Cauchy sequence.

Solution

Let $\left\langle a_{n}\right\rangle$ is a convergent sequence with $b$.

$$
\begin{aligned}
& \forall \varepsilon>0, \exists n_{o} \in N: n>n_{0} \Rightarrow\left|a_{n}-b\right|<\frac{\varepsilon}{2} \text {. } \\
& m>n_{0} \Rightarrow\left|a_{m}-b\right|<\frac{\varepsilon}{2} . \\
& \text { For } \quad n, m>n_{0} \Rightarrow\left|a_{n}-a_{m}\right| \\
& =\left|a_{n}-b+b-a_{m}\right| \\
& \leq\left|a_{n}-b\right|+\left|b-a_{m}\right| \\
& <\frac{\varepsilon}{2}+\frac{\varepsilon}{2} \\
& =\varepsilon \text {. }
\end{aligned}
$$

Then $\left\langle a_{n}\right\rangle$ is a Cauchy sequence.

\subsubsection{Example}

Show that every Cauchy sequence $\left\langle a_{n}\right\rangle$ of real numbers is bounded.

\section{Solution}

Let $\left\langle a_{n}\right\rangle$ be a Cauchy sequence.

$$
\begin{gathered}
\text { Let } \varepsilon=1, \exists n_{o} \in N: n, m>n_{0} \Rightarrow\left|a_{n}-a_{m}\right|<1 . \\
m \geq n_{0} \Rightarrow\left|a_{n}-a_{m}\right|<1 . \\
\text { i.e, } \quad-1<a_{n}-a_{n_{0}}<1 \\
a_{n_{0}}-1<a_{n}<a_{n_{0}}+1
\end{gathered}
$$

Let $\propto=\max \left(a_{1}, a_{2}, \ldots, a_{n_{0}}, a_{n_{0}}+1\right)$

$\beta=\min \left(a_{1}, a_{2}, \ldots, a_{n_{0}}, a_{n_{0}}-1\right)$.

Then $\alpha$ is an upper bound and $\beta$ is a lower bound for the range $\left\{a_{n}\right\}$ of the sequence $\left\langle a_{n}\right\rangle$. Hence $\left\langle a_{n}\right\rangle$ is bounded.

\subsection{Some Properties on Topological Spaces}

Let $X$ be a non-empty set. The class $\tau$ of subsets of $X$ is a topology on $X$ if and only if $\tau$ satisfies the following axioms.

$\left[O_{1}\right] \quad \varnothing \in \tau, X \in \tau$.

$\left[\mathrm{O}_{2}\right]$ The union of any number of sets in $\tau$ belongs to $\tau$.

$\left[O_{3}\right]$ The intersection of any two sets in belongs to $\tau$.

The numbers of $\tau$ are called open sets and the pair $(X, \tau)$ is called a topological space [1-3].

\subsubsection{Usual Topology}

Let $\mu$ denote the class of all open sets of real numbers. Then $\mu$ is a topology on $R$, called usual topology on $R$.

\subsubsection{Example}

Consider the classes of subsets of $X=\{a, b, c, d, e\}$.

$\tau_{1}=\{X, \emptyset,\{a\},\{c, d\},\{a, c, d\},\{b, c, d, e\}\}$

$\tau_{2}=\{X, \varnothing,\{a\},\{c, d\},\{a, c, d\},\{b, c, d\}\}$.

$\tau_{3}=\{X, \emptyset,\{a\},\{c, d\},\{a, c, d\},\{a, b, d, e\}\}$.

Solution

Observe that $\tau$ is a topology on $X$, since it satisfies the three axioms.

For, since $X$ and $\emptyset$ belong to $\tau_{1}$, the axiom $\left[O_{1}\right]$ is satisfied.

Since the union of any number of sets in $\tau_{1}$ belongs to $\tau_{1}$, the axiom $\left[O_{2}\right]$ is satisfied.

Since the intersection of any two sets in $\tau_{1}$ belongs to $\tau_{1}$, the axiom $\left[O_{3}\right]$ is satisfied.

For $\tau_{2}, \tau_{2}$ is not a topology on $X$, since $\{a, c, d\} \in \tau_{2},\{b, c, d\} \in \tau_{2}$, but $\{a, c, d\} \cup\{b, c, d\}=\{a, b, c, d\} \notin \tau_{2}$, i.e, $\tau_{2}$ does not satisfy the axiom $\left[\mathrm{O}_{2}\right]$

For $\tau_{3}, \tau_{3}$ is not a topology on $X$, since $\{a, c, d\} \in \tau_{3},\{a, b, d, e\} \in \tau_{3}$, but $\{a, c, d\} \cap\{a, b, d, e\}=\{a, d\} \notin \tau_{3}, i . e ., \tau_{3}$ does not satisfy the axiom $\left[O_{3}\right]$.

\subsection{Discrete Topological Space and Indiscrete Topological Space}

Let $D$ be denote the class of all subsets of $X$. Then $D$ satisfies the axioms for a topology on $X$. This topology is called the discrete topology and $(X, D)$ is called a discrete topological space.

The class $J=\{X, \emptyset\}$ is a topology. It is called the indiscrete topology and $(X, J)$ is called a indiscrete topological space [4]. 
International Journal of Advances in Scientific Research and Engineering (ijasre), Vol 5 (12), December-2019

\subsubsection{Example}

The intersection $\tau_{1} \cap \tau_{2}$ of any two topologies $\tau_{1}$ and $\tau_{2}$ on $X$ is also a topology on $X$.

Solution

Since $\tau_{1}$ and $\tau_{2}$ are topologies on $X, X$ and $\emptyset$ each belongs to both $\tau_{1}$ and $\tau_{2}$.

Hence $X$ and $\emptyset$ each belongs to $\tau_{1} \cap \tau_{2}$. The axiom $\left[O_{1}\right]$ is satisfied.

Let $A_{i} \in \tau_{1} \cap \tau_{2}$, then $A_{i} \in \tau_{1}$ and $A_{i} \in \tau_{2}$ for every $i$. Since $\tau_{1}$ and $\tau_{2}$ are topologies, $\bigcup_{i} A_{i} \in \tau_{1}$ and $\bigcup_{i} A_{i} \in \tau_{2}$.

Then $\bigcup_{i} A_{i} \in \tau_{1} \cap \tau_{2}$. The axiom $\left[O_{2}\right]$ is satisfied.

If $G, H \in \tau_{1} \cap \tau_{2}$, then $G, H \in \tau_{1}$ and $G, H \in \tau_{2}$. Since $\tau_{1}$ and $\tau_{2}$ are topologies, $G \cap H \in \tau_{1}$ and $G \cap H \in \tau_{2}$.

Then $G \cap H \in \tau_{1} \cap \tau_{2}$. The axiom [ $\left[O_{3}\right]$ is satisfied. Thus $\tau_{1} \cap \tau_{2}$ is a topology on $X$.

But, the union of topologies need not be a topology.

Counter example,

$X=\{a, b, c\}, \tau_{1}=\{X, \emptyset,\{a\}\}, \tau_{2}=\{X, \emptyset,\{b\}\}$.

$\tau_{1}$ and $\tau_{2}$ are topologies.

$\tau_{1} \cup \tau_{2}=\{X, \emptyset,\{a\},\{b\}\}$.

$\{a\} \in \tau_{1} \cup \tau_{2}$

$\{b\} \in \tau_{1} \cup \tau_{2}$

$\{a\} \cup\{b\}=\{a, b\} \notin \tau_{1} \cup \tau_{2}$

Therefore $\left[\mathrm{O}_{2}\right]$ is not satisfied.

Therefore $\tau_{1} \cup \tau_{2}$ is not a topology.

In topology, a discrete space is a particularly simple example of a topological space or similar structure, one in which the points form a discontinuous sequence, meaning they are isolated from each other in a certain sense. The discrete topology is the finest topology that can be given on a set, i.e., it defines all subsets as open sets. In particular, each singleton is an open set in the discrete topology [5].

\section{CONCLUSIONS}

We have discussed the basic properties open sets, closed sets and some characterizations of these sets on the topology of the line and plane. Moreover, it is also mentioned in this paper that a topological spaces. Some illustrative examples have been explored in order to distinguish between their properties. It has been vividly discussed in this paper. I hope thatthese notes help; please do let me know if anything requires clarification.

\section{ACKNOWLEDGEMENT}

I would like express my sincere gratitude to Reactor, Dr. Kay Thi Lwin, Technological University (Hmawbi) for her encouragement to carry out this paper. I am grateful thanks to Dr. Zin Mar Htet, Department of mathematics, Bago, University, who devoted a great deal of time to supervising with great patience in the preparation of this paper.

\section{REFERENCES}

[1] S.Lipschutz, "Theory and Problem of General Topology", McGraw-Hill, 1965.

[2] Huy"nh Quang Vu, "Lecture notes on Topology", Version of January 26, 2018.

[3] Renzo's Math 490, "Introduction to Topology", Winter 2007.

[4] Xin-She Yang, "Engineering Mathematics with Examples and Applications", Middlesex University, School of Science and Technology.

[5] J.McCleary, "A First Course in Topology", Student Mathematical Library, Volume 31, 2006.

[6] W.F.Basener, "Topology and Applications", Wiley-Interscience, 2006. 